\title{
Dental Biometrics: Human Identification Based On Dental Work Information
}

\author{
Michael Hofer ${ }^{1}$, Aparecido Nilceu Marana ${ }^{2}$ \\ ${ }^{1}$ Medical Information Technology, Carinthia University of Applied Sciences, Klagenfurt, Austria \\ ${ }^{2}$ UNESP, Faculdade de Ciências, Departamento de Computação, Bauru - SP, Brazil \\ m0399homi@fh-kaernten.at -nilceu@fc.unesp.br
}

\begin{abstract}
Dental biometrics is used in forensic dentistry to identify or verify persons based on their dental radiographs. This paper presents a method for human identification based on dental work information. The proposed method works with three main processing steps: segmentation (feature extraction), creation of a dental code, and matching. In the segmentation step, seed points of the dental works are detected by thresholding. The final segmentation is obtained with a snake (active contour) algorithm. The dental code is defined from the position (upper or lower), the size of the dental works, and distance between neighboring dental works. The matching stage is performed with the Edit distance (Levenshtein distance). The costs for the insertion, deletion and substitution operations were adapted to make the matching algorithm more sensitive. The method was tested on a database including 68 dental radiographs and the results are encouraging.
\end{abstract}

\section{Introduction}

Biometrics is the science and technology of identification, i.e. establishing the identity of an individual, by measuring the subject's physical or behavioral traits. The term is derived from the Greek words "bios" for life and "metron" for to measure [1]. The method of dental biometrics is used in forensic medicine (forensic dentistry) to identify persons by matching post-mortem radiographs (acquired after a person is deceased), with ante-mortem radiographs (acquired before a person is deceased) in a database, but can also be used to match two ante-mortem or two postmortem radiographs (see Fig. 1). In some cases (plane crashes, fire accidents, etc.) biometric features such as faces or fingerprints are destroyed and it is not possible to work with conventional identification methods like fingerprint or face recognition. In such cases, dental biometric is an appropriated method, because bones and teeth with their dental works (DWs), e.g. inlays, are very resistant to modest force effects and high temperatures (amalgam fillings up to $1000^{\circ} \mathrm{C}$, endodontic treatments up to $1100^{\circ} \mathrm{C}$ [2]) and also posses good biometric properties. Dental records have been used to identify the victims of disasters, such as the $9 / 11$ bombing and the Asian tsunami $[3,4]$.

The objective of this work is to develop and implement a dental biometric method for human identification based on DW information. The algorithm performs DW matching onto registrated panoramic dental radiographs and is implemented in Matlab ${ }^{\mathrm{TM}}$.

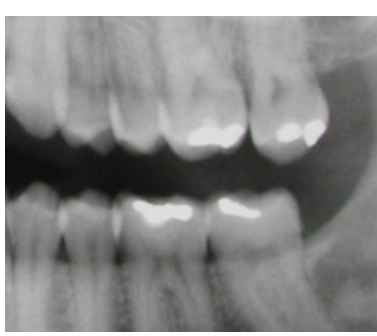

(a)

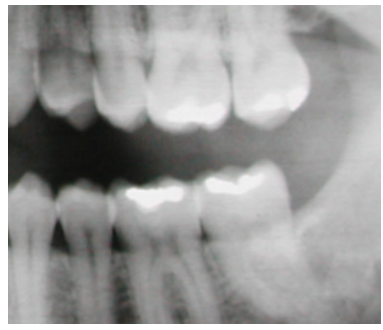

(b)
Figure 1: AM radiographs of the same person. (a) Acquired in the year 2000, (b) Acquired in the year 2003. High dental work similarity in both images can be observed.

\section{Methods}

The proposed method for human identification based on DW information consists of three main processing steps:

1. Pre-processing of the dental radiographs (DRs) and segmentation of the DWs.

2. Creation of a "dental code" (DC) out of the information of the detected DWs including 
position (upper or lower jaw), size and distance between neighboring DWs.

3. Matching of a particular DC with other DCs in a database.

\section{Step 1: Pre-processing and segmentation}

The DR (RGB image) is converted into a gray-scale image and median filtering (size 6x6) is performed to reduce noise in the image. Because of different lightning conditions in the DRs, the image is subdivided into two regions of interest (ROIs): left (ROI 1) and right (ROI 2), see Fig. 2.

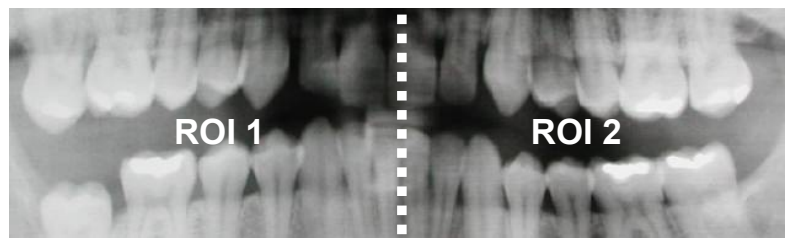

Figure 2: ROls in a dental radiograph, left part (ROI 1), right part (ROI 2).

The algorithm determines a gray value threshold in the left and the right ROI of the DR. Typically the DWs feature the highest intensities in the image and appear as a distinct, relatively small but pronounced, mode in the upper range of the gray-scale histogram. After smoothing the histogram with a moving average filter, the threshold is set to the gray-value at the location of the left valley at the rightmost mode, which indicates the DWs (see Fig. 3).

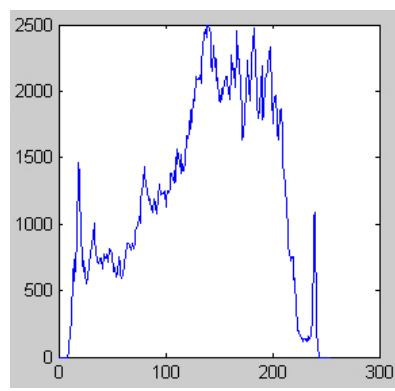

(a)

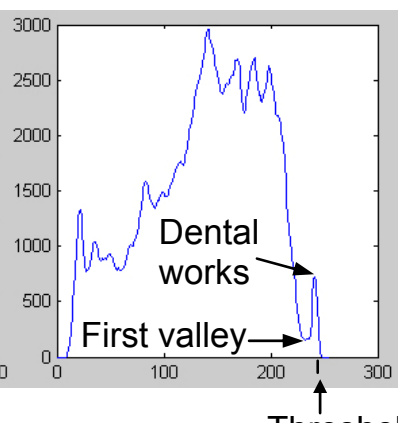

(b)
Threshold

Figure 3: (a) Histogram of the ROI 2 in image Fig. 2, (b) Smoothed version of the histogram with detected threshold (threshold value is 232).

The threshold is used to binarize the gray-value image (see Fig 4.). The results of the conversion are used as initial contours for the segmentation stage. Each region represents a possible DW.

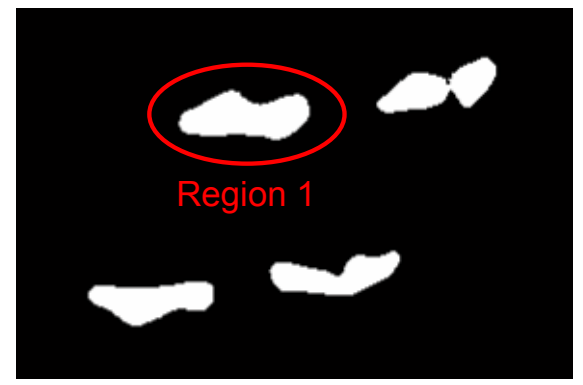

Figure 4: Binary image (ROI 2 in image Fig. 2) including possible dental works, e.g. region 1.

A snake (active contour) algorithm [5] is used to perform the final segmentation of the DWs. Snakes can be used to segment objects with fuzzy border contours where traditional edge-detection [6] will fail. Snakes are curves that can move under the influence of internal forces (elasticity and bending forces) coming from within the curve itself and external forces (potential forces) computed from the image data. The internal and external forces are defined so that the final snake will conform to an object boundary [5]. The external force field is computed from the gradient image, as shown in Fig. 5. A snake needs to be initialized with an initial curve (e.g. circle) and is an iterative procedure which stops after a defined number of iterations. The better the initialization curve, the better the performance of the algorithm and the final segmentation results.

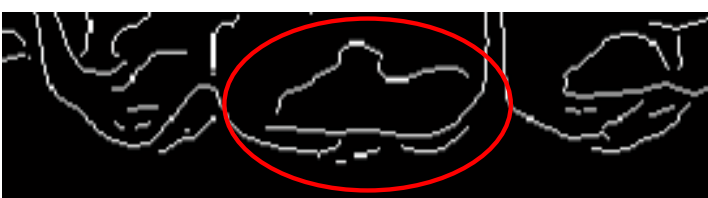

(a)

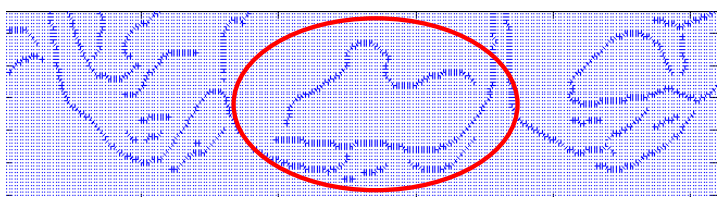

Figure 5: (a) Edge map of the intensity image, (b) Gradient of the edge map. The areas in the circles are equal to region 1 in Fig. 4.

Each DW is segmented with a separate snake. To improve the segmentation and to speed up the algorithm, the initial curves for all DWs are computed from the binary mask. The borders of the detected regions are used as initial curves (see Fig. 6). The 
evaluation of the snake method for the final segmentation of a DW contour is shown in Fig. 7.

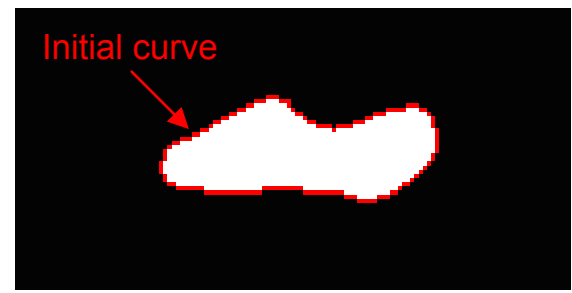

Figure 6: Initial curve of a snake computed out of the binary image. The border of the region represents the initial curve (region 1 in Fig. 4).

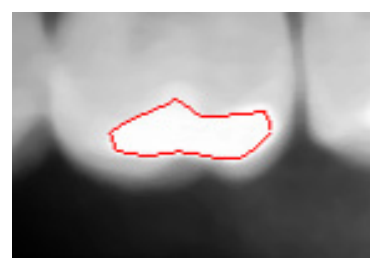

(a)

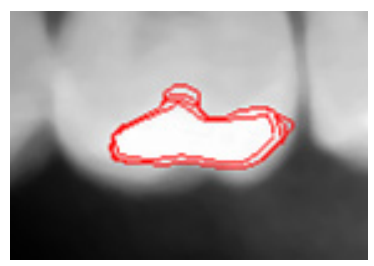

(c)

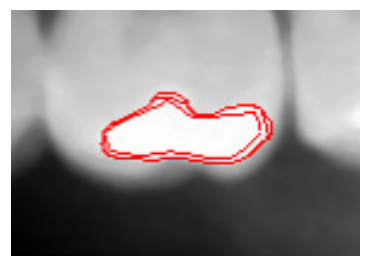

(b)

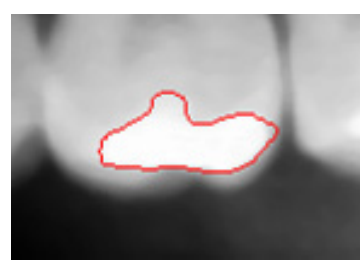

(d)
Figure 7: Segmentation process (region 1 in Fig. 4). (a) Initial curve, (b) Curve transformation after 2 iterations, (c) After 5 iterations, (d) Final segmentation result after 30 iterations.

Finally, a binary mask of the image, including all detected DWs, is created, which is called dental works mask (DWM).

\section{Step 2: Creation of the dental code}

Based on the DWM, a dental code (DC) is created. The DC incorporates information about the position (upper or lower jaw), the size of the DWs and the distance between two neighboring DWs.

\section{Location of the dental work}

An algorithm was implemented to sort all DWs in the DWM from left to right based on the center of mass point of each individual DW (see Fig. 8).

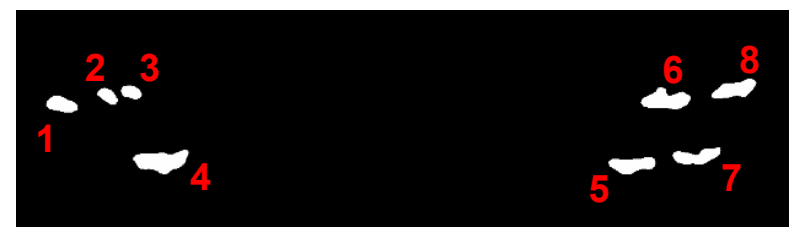

Figure 8: Dental work mask with sorted dental works from left to right.

For the DC it is also important to know whether the tooth, where the DW is located, belongs to the maxilla (upper jaw) or to the mandible (lower jaw). Therefore, a border between the maxillary and the mandibular teeth is detected. A stripe in the intensity image is cut with the width of the current region. Next, the intensity sum of all horizontal rows in the stripe is calculated. The highest intensity represents the area of the DW (see Fig. 9). The algorithm detects the first valley on the left and on the right site of the highest intensity point. The valley with the lower intensity represents the border between the mandibular and maxillary teeth. If the position of this valley is above the DW in the image, the DW belongs to the mandible ("L"). If the position is below the DW, the DW belongs to the maxilla ("U") (see Fig. 9). The location of the DW is represented in the DC with the letter " $\mathrm{L}$ " or " $U$ ".

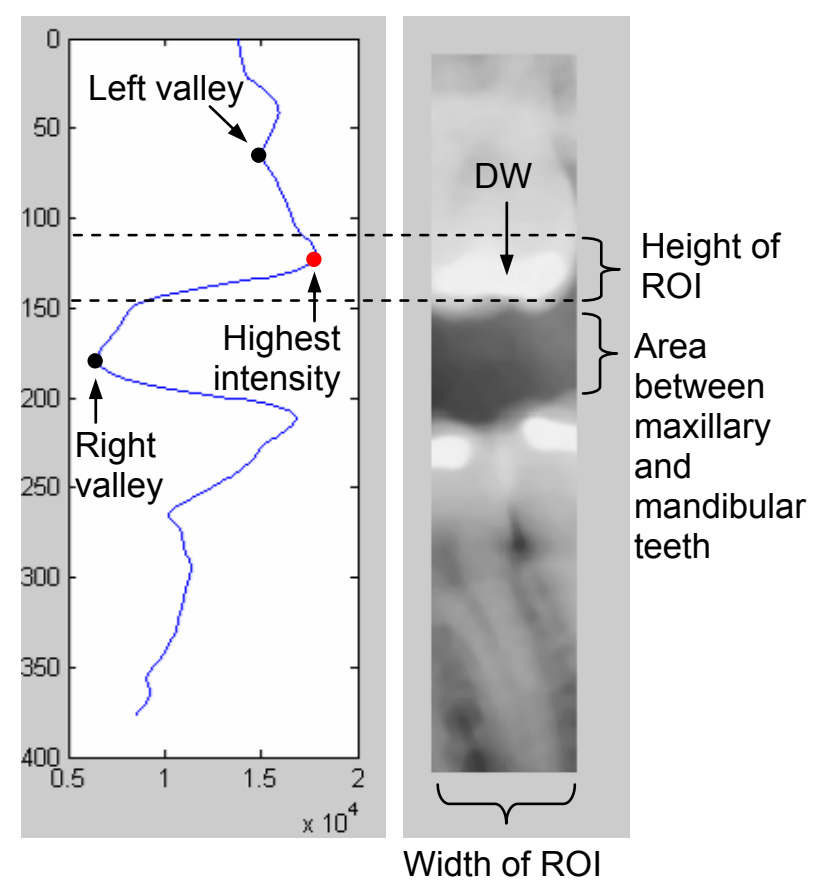

Figure 9: Cut stripe (region 1 in Fig. 4) and sum of intensities; right valley represents lower intensity which indicates that the DW belongs to the maxilla teeth (dental code = "U”). 


\section{Size of the dental restoration}

The proposed method uses registrated DRs. Useful image information, including mandibular and maxillary teeth, is cut and resized to a size of $1000 \times 300$ pixels. According to this, the amount of pixels in a DR is always the same, which means that the size of a DW (amount of pixels) is a percentage of the total amount of pixels in the DR. To have a better overview in the DC, the size is multiplied by $10^{3}$ (see Fig. 11).

\section{Distance between two dental works}

To make the matching algorithm more sensitive, also the distance (amount of pixels) between two neighboring DWs is included into the DC. The distance is defined by the amount of pixels between the center of mass points of the two DWs. The distance of the leftmost DW (d1) is set to zero to make the algorithm more stable against small deviations in the manually registration of the DRs (see Fig. 10). The value for the distance is given in percentage of the total width of the DR, which is always 1000 pixels. To have a better overview in the DC, the distance is multiplied by $10^{2}$.

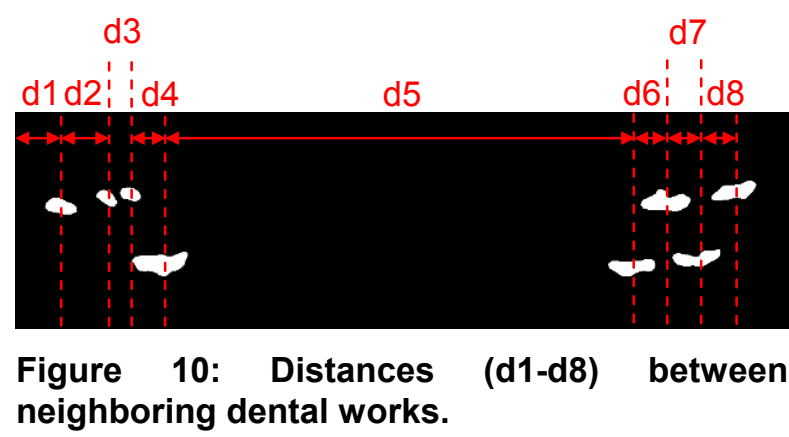

Out of this information, the DC is built as follows: At first the location of the DW ("L" or "U"). At second the size of the DWs, and at third the distance between the current DW and the left neighbour DW. An example of a final DC for a DR is shown in Fig. 11.

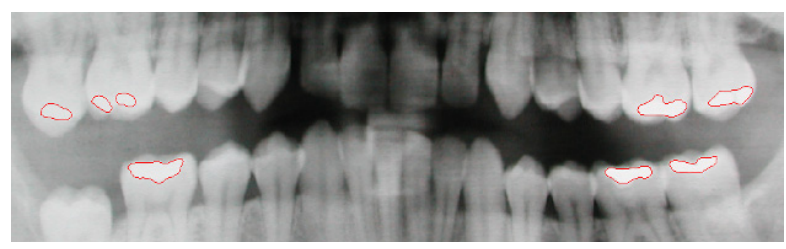

DC: “U2.1-0_U1.207-6_U1.187-3_L4.773-3.9_L3.06360.6_U3.893-4.4_L2.86-4_U3.093-4.9_"

Figure 11: Final dental code of a dental radiograph.

\section{Step 3: Matching}

After the DC is created, it can be compared to other DCs in a database. These can be different codes of the same person or codes of different persons. Matching between radiographs of the same subject is called "genuine matching" and matching between radiographs belonging to different subjects is called "impostor matching".

An algorithm was implemented which works with the "Edit distance" (Levenshtein distance). The Edit distance is often used to compare gene sequences or strings. The Edit distance between two strings is given by the minimum number of operations needed to transform one string into the other, where an operation is an insertion, deletion, or substitution [7]. Every operation is associated with certain costs. Because of the structure of the DC, it was necessary to restructure the algorithm of the Edit distance. Not only the letters "U" and "L" have to be compared, but also the size of the DWs and the distance between neighboring DWs. The costs of the operations insertion, deletion, or substitution were adapted to improve the results of the matching algorithm. In the case of insertion and deletion, the matching cost is 60 (see Tab. 1). In the case of substitution, the cost for comparing two DWs is given by the sum of two costs: (i) the cost of comparing the size and (ii) the cost of comparing the distance of two DWs.

If the compared sizes differs more than $100 \%$, the cost is set to 25. In the other case, the cost for comparing the size is set according to the percentage difference of the two compared DWs (see Tab. 1).

If the compared distances differs more than $15 \%$, the cost is set to 25 . In the other case, the cost for comparing the distances is set according to the percentage difference of the two compared DWs (see Tab. 1). 
Table 1: Costs for insertion, deletion and substitution in the Edit distance.

\begin{tabular}{|c|c|}
\hline Operation & Cost \\
\hline insertion & 60 \\
\hline deletion & 60 \\
\hline substitution & 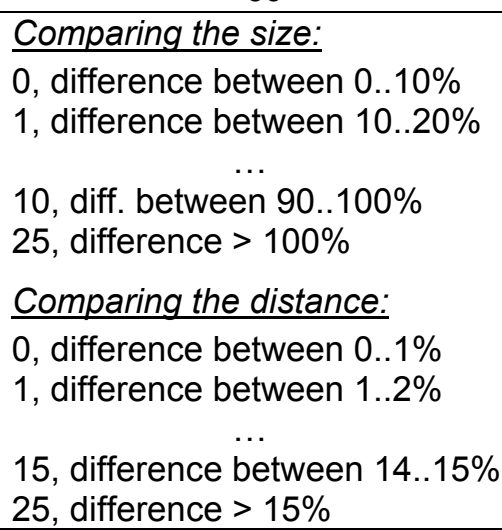 \\
\hline
\end{tabular}

\section{Results}

A database including 68 DRs was used in the experiments to evaluate the proposed dental biometric method: a pair of DRs for 22 subjects (44 radiographs) plus a single DR for other 24 subjects. For the 22 subjects with two radiographs, their oldest DRs were considered as AM (ante-mortem) radiographs and their newest DRs were considered as PM (post-mortem) radiographs. For the 24 subjects with only one radiograph, the DRs were considered as AM radiographs. The images were manually registrated to obtain comparable conditions. In cases of over- or under segmentation of the DWs, the segmentation result had to be corrected manually (see Fig. 12). Also, if DWs are not detected by thresholding, a ROI has to be selected manually in the DR to perform local thresholding. Segmentation results of two distinct DRs and their corresponding DCs are shown in Fig. 13.

To test the matching performance of the method, an algorithm was implemented to compare DRs of the genuine class (two radiographs of the same person) and DRs of the impostor class (two radiographs of different persons). Figure 14 shows a receiver operating characteristic (ROC) curve, which plots the false acceptance rate (FAR) versus the false rejection rate (FRR) for different threshold values. It is possible to observe in the ROC curve that the proposed method obtained $11 \%$ of equal error rate (EER) for the used database, a good result. The equal error rate (EER) is the value where the FAR is equal to the FRR. The lower the EER, the better is the performance of the biometric system $[8,9]$.

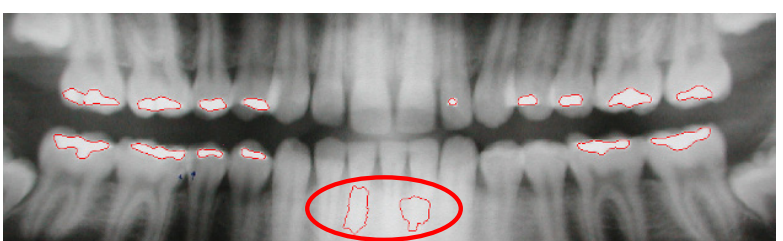

Figure 12: Example of over-segmentation (areas in the circle).

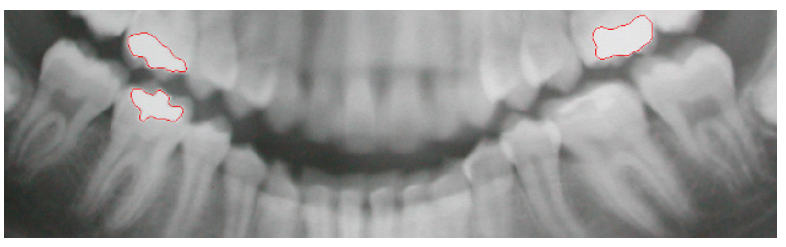

DC: “U6.997-19.5_L6.147-0.1_U9.523-60_"

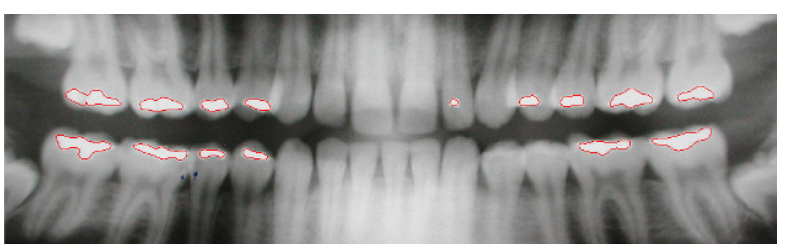

DC: "L3.877-10.1_U3.357-1.2_U2.543-8.9_L2.57-

0.1_L0.947-6.5_U1.453-0.5_L0.953-5.1_U1.233-

0.5_U0.273-25. 2 U $0.937-9.7$ U1.287-5.6_L2.67-

4.1_U2.927-3.5_L3.733-6.8_U1.913-1.6_"

Figure 13: Segmentation results of two dental radiographs of different subjects including their dental codes.

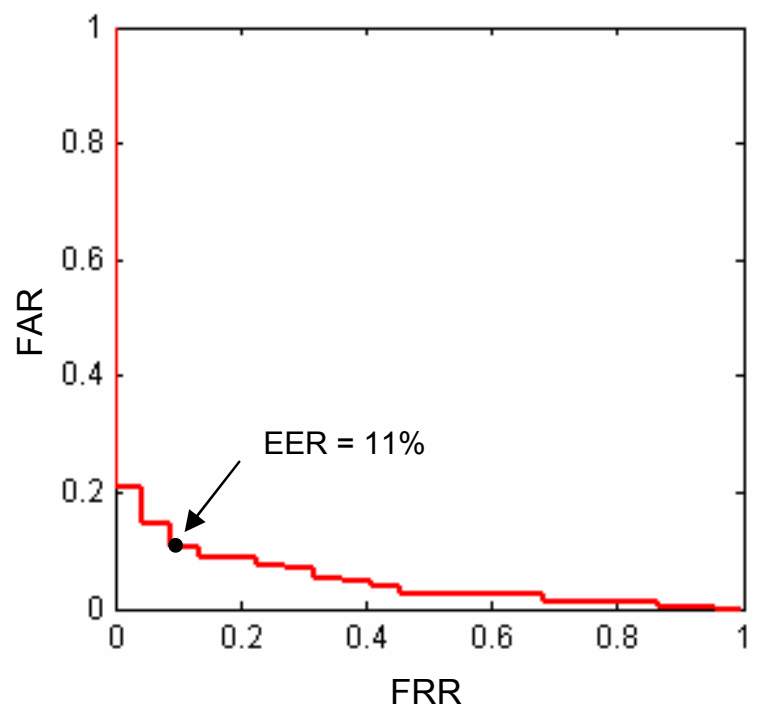

Figure 14: ROC curve, input data: 44 DRs (22 pairs of genuine DRs). EER is $11 \%$. 
Figure 15 shows the accuracy curve obtained when the $22 \mathrm{PM}$ radiographs were matched to the $46 \mathrm{AM}$ radiographs of the database. Using the top-1 retrieval, the accuracy was $19 / 22 \quad(=86 \%)$. Using top- 8 retrievals, the retrieving accuracy was $95 \%$. The accuracy reached $100 \%$ when the top- 11 retrievals were used.

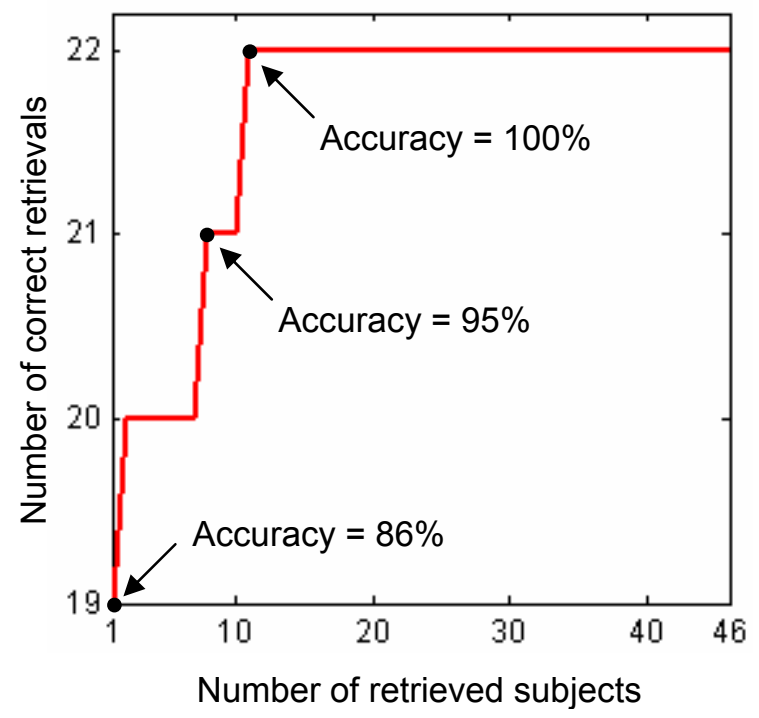

Figure 15: Accuracy curve obtained when 22 subjects were identified from 46 possible identities.

In our experiments, the proposed segmentation method performed accurate segmentation onto good quality DRs. If the quality of the DR was not good, the automatic segmentation failed and some human intervention had to be done.

\section{Conclusion and Future Work}

Dental biometrics is used to identify individuals in the forensic medicine. This paper presents a dental biometric identification method based only on dental work information extracted out of panoramic DRs.

For the database used in the experiments, the results obtained were good (11\% of EER and $86 \%$ of accuracy for top-1 retrieval), especially considering that only dental work information is considered by the method.

Because the amount of images in the database is low, it is not possible to make a clear statement about the performance and effectiveness of the proposed method. In future work, the proposed method will be assessed on a larger database. Also, in future work, the proposed method will be fused to other methods based on different tooth features, like crown and root shapes, and the segmentation method will be replaced by a method based on image-foresting transform, more effective for segmentation of bad quality images, with minimum human intervention.

\section{Acknowledgement}

The authors are grateful to Dr Izabel Regina Fischer Rubira-Bullen, Bauru Dental School, University of Sao Paulo, for providing most of the dental radiographs.

\section{References}

[1] National Science and Technology Council (NSTC) Subcommittee on Biometrics. "Introduction to Biometrics." Internet: http://www.biometrics.gov/, [May 14, 2007].

[2] C. Savio, G. Merlati, P. Danesino, G. Fassina, P. Menghini. "Radiographic evaluation of teeth subjected to high temperatures: Experimental study to aid identification processes" Forensic Science International; vol. 158(2); pp. 108-116, May 2006.

[3] P. O'Shaughnessy. "More than Half of Victims IDd." Internet:

http://911 research.wtc7.net/cache/planes/evidence/dailynews _halfvictimsidd.html, Sept. 11, 2002 [Feb. 23, 2007].

[4] H. Chen, A.K. Jain. "Dental Biometrics: Alignment and Matching of Dental Radiographs." IEEE Trans. PAMI; vol. 27(8); pp. 1319-1326, August 2005.

[5] X. Chenyang, J. L. Prince. "Snakes, Shapes, and Gradient Vector Flow." IEEE Trans. on Image Processing, vol 7(3), pp. 359-369, March 1998.

[6] D. Ziou, S. Tabbone. "Edge Detection Techniques - An Overview." International Journal of Pattern Recognition and Image Analysis; vol. 8; pp. 537-559, 1998.

[7] National Institute of Standards and Technology, "Levenshtein Distance." Internet: http://www.nist.gov/dads/HTML/Levenshtein.html, Nov. 10, 2005 [Feb. 23, 2007].

[8] HumanScan GmbH. "About FAR, FRR and EER." Internet: http://www.bioid.com/sdk/docs/About_EER.htm, March 3, 2007 [Jan. 28, 2007].

[9] D. Maltoni, D. Maio, Anil K. Jain, Salil Prabhakar, "Handbook of Fingerprint Recognition.", Springer-Verlag, New York, USA, 2003. 